From the Pediatric Department Keio University, School of Medicine Shinjuku-ku, Tokyo, Japan

\title{
The Adrenocortical Function Studies in Children
}

\section{$b y$}

IWAO TAKAKURA

\begin{abstract}
The recent advances in the laboratory procadures make it possible to study endocrinological staties in childnen more easily and more accurately even in the neonatal period. The successes in purification and the determination of chemical structures of various hormones and their releasing factors make it possible to carry out various loading tests. The progress in the measurement of various hormones and their metabolites especially in the field of radioimmumoassuy is really remarlabble.

If we took adrenocortical function studies as an example, urinary 17 hydroxycorticosteroid was the only reliable item about ten ycars ago, then plasma 17-OHCS determination using Porter-Sitver chromogen was introduced followed by plasma 11-OHCS determination with fluonesconce spectrophotometry. Then, cortisol production rate determination became possible using radio-isotope dilution technique, and at present, plasma cortisol can be measured directly with protein competitive nadio-immunoassay. Thus, the adrenocortical function studies in the pediatric age group has become easier and mone noliable. The author likes to present some endocrinologiaxl nesults in children using various methods and now they can be applited in the clinitial work.
\end{abstract}

Invited Paper at the Third National Indonesian Pediatric Congress, Surabaya, July $1-6,1974$ 
The pediatric endocrinology has advanced remarkably in recent years. This advance is due very much to the development of new techniques such as radioimmunoassay. Even the neonates who could not be studied endocrinologically because of the difficulties in obtaining the blood or urine samples in sufficient quantity, can be studied with less difficulty at present. Many hormones and their metabolites that could not be isolated or determined before, can now be determined and many new findings have been reported. Thus, pediatric endocrimology is being divided into finer and finer subspecialties. Therefore, it is really impossible for a single person with limited time to review all the new advances and I would like to limit myself to talk only on the adrenocortical function studies in children, particularly on glucocorticoids.

The physiological significance of the adrenals started to be appreciated as a consequence of the description by Addison in 1855 . He reported the clinical syndrome known as Addison's disease resulting from destructive disease of the adrenal glands. Brown Séquard concluded that the adrenal glands are essential for life after the experiments on the effect of adrenalectomy in 1856. Around 1920 it was generally conceded that the cortex rather than the medulla was the life-mainitaining portion of the gland. Thereafter, numerous physiological abnormalities started to be exhibited by adrenalectomized animals and could be corrected by adrenocortical extracts. The preparation of extracts with a reasonable degree of biological activity was first accomplished in 1930, then the chemical identification and synthesis have been developed.

Meanwhille, Foster and Smith in 1926 reported the fact that hypophysectomy results in atrophy of the adrenal cortex. This was the beginning of the studies on the adrenocorticotropic hormone, the so-called ACTH. The chemical isolation and the purification of ACTH of the hypophyses had developed by 1952 and the structure of am ACTH polypeptide was unravelled by Belli and associate workers in 1956 and it can be synthesized at present.

Various methods have been tried so far as tests of adrenocortical function, but after the introduction of ACIT, the adremocortical reserve. function tests, using exogenous ACTH as a stimulating factor, became the most important and widely applied methods. The most popular test when the adrenocortical hormones or their metabolites in blood or in urine could not be determined directly was the socalled Thorn test. Naturally, this test could not be a reliable indicator of adrenocortical function because only the changes of 
eosinophils in blood could be studied in the test.

Urimary 17-OHCS is the first clinically used corticosteroid metabolite for the adrenocortical function lest. Principal products of metabolic transformations of cortisol recovered from urine as 17-OHCS are tetrahydrocortisol and tetrahydrocontisone. The method of determination and some results will be explained by slides. As for the reserve function test, exogenous ACTH is administered. This test has some disadvantages when it is applied in children. Those are: (1) Difficulties in collecting urine accurately (2) Single voiding specimen can not be an indicator and 24 hour urine collection is necessary (3) Three days are needed to collect urine for the reserve function test and more than 24 hours are needed for the determination procedures.

Plasma 17-OHCS determination comes next. The method of the test using plasma 17-OHCS as and indicator will also be explained by slides. This test has also some disadvanta. ges. The biggest is that a relatively large amount of blood for children is needed for the determination.

Plasma 11-OHCS is a fainly ideal adrenocortical reserve function test for children, in contrast with the many disadvantages of the above mentioned methods. The method and some results of this test will also be explained by slides. Because of the clinical application of synthetic ACTH, the so-called rapid test using ACTH intravenously has become possible to perform without fearing the incidence of anaphylactic reaction due to ACTH of animal origin. The test can be performed even at the outpatient department. The actual duration of test is only about one hour and the determination procedure itself is short and can be finished within one hour. The amount of blood needed for the determination is less than that for plasma 17OHCS determination and not sodifficult to obtain, even from infants. The results of this test in pediatric cholera patients may be interesting and it will be shown in slides. The conclusion is, that even in circulatory shock these cholera patients had enough reserve of adrenocortical function and could show a good response to ACTH administration. Therefore, it is not effective to give ACTH or adrenocorticosteroid therapy to those cholera patients.

All those tests mentioned above represent the adrenocortical reserve function to stimuli such as exogenous ACTH. The adrenocortical function in normal state without stimuli can be represented by the cortisol production rate. The determination of cortisol production rate, 
being called CPR in a briged form, can now be performed due to the recent advances in the field of isotope techniques. The method will also be explained on slides. This test is useful in the evaluation of adrenocortical insufficiency, such as Addison's disease and congenital adrenal hyperplasia.

Meanwhile, the tests being aimed at for determination of hypophyseal function regarding the release of endogenous ACTH have also been developed. One of them is the socalled Metopirone or Metyrapone test. This substance inhibits the 11B-hydroxylation of the steroid biosynthesis. Because of this inhibitory action, plasma 11-desoxycortisol cannot be converted to cortisol, causing the plasma cortisol level to drop. This drop stimulates the hypophysis to release ACTH. Any of the plasma ACTH, urinary 17-OHCS and urinary 17-KGS can be indicators to know the degree of this inhibitory action but usually urinary 17-OHCS is determined as the indicator becauge the determination procedure is the easiest among them and still reliable enough. The method and some of the results will also be explained by slides. Plasma ACTH can be determined directly at present by radioimmunoassay. Those tests make it possible to diffenentiate hypophyseal dysfunction and ad- renocortical insufficiency when the patients have clinical signs of adrenocortical insufficiency.

Adrenocorticosteroid therapy is now widely used in the pediatric practice. As the release of ACTH from the hypophysis is regulated by plasma cortisol concentration, the administration of corticasteroid tends to cause adrenocortical suppression. (The findings on this subject will be explained by slides. In short, dexamethasone suppresses the adrenal cortex more in comparison with other forms of synthetic corticosteroid preparation. During corticosteroid therapy, ACTH administration is not effective to correct this suppression. The recovery from this suppression can be expected within a no't so long period after the discontinuation of the corticosteroild therapy in the majority of cases.

Congenital adrenal hyperplasia, particularly, the salt-losing type, is a lifethreatening problem. Fortunately, because of the advances in diagnostic procedure, as stated already, it is not so difficult to diagnose those patilents, even in the neonatal period, and they can be treated adequately utilizing the data obtained by above mentioned adrenocortical function studies such as CPR.

In recent years, most of the adrenocortical function studies are per- 
formed in the neonatal period and dings in neonatal endocrinology have many new findings are being repor- been reported already, but still many ted. Aside from glucocorticoid, problems remain unsolved. We shall aldosterone can be secreted by the look forward to the progress in this fetus and salt regulation can be field because it will be a great benedone as in adults, even in preterm fit for the children and for the babies. Many other interesting fin- people. 